\title{
A CORRECTED $T(q)$-LIKELIHOOD ESTIMATOR FOR THE EXPONENTIAL STRUCTURAL MEASUREMENT ERROR MODEL
}

UDC 519.21

\author{
A. V. SAVCHENKO
}

\begin{abstract}
The exponential structural measurement error regression model is studied. The corrected $T(q)$-likelihood estimator of regression coefficients is constructed. A sufficient condition for the strong consistency of the estimator is presented for the case where the sample size tends to infinity, $q$ depends on the sample size, and $q \rightarrow 1$.
\end{abstract}

\section{INTRODUCTION}

A general nonlinear regression model with measurement errors is studied in the paper. We assume that the conditional distribution with respect to the hidden variable in the model is exponential.

If the distribution of the hidden variable is unknown, then the so-called corrected score procedure yields a consistent estimator; see [4. It is also known that the corrected score estimator is unstable if the size of a sample is small or moderate. A modification of the corrected score estimator is constructed in the papers [1] and [5] for small samples. This modification is more stable for a small or moderate size of a sample. Moreover, this estimator is asymptotically equivalent to the corrected score estimator as the size of a sample tends to infinity.

Another modification of the corrected score estimator for a small and moderate size of a sample is constructed in the current paper. Our approach is different from that in [1] and [5].

There are a number of papers related to the so-called $T(q)$-likelihood estimator for the case where there is no measurement error in the variables. Some properties of the $T(q)$-likelihood estimator are studied in the papers [2, 3. by using the methods of the asymptotic analysis and computer simulation.

The aim of the current paper is to consider a corrected $T(q)$-likelihood estimator for the measurement error regression model.

By the same symbol $\mathrm{E}$ we denote the expectation of random variables, vectors, or matrices, while Var stands for the variance. The expectation $\mathrm{E}_{b} f$ is considered under the condition that $b$ is the true value of the parameter $\beta$. The upper index $T$ means the transposition.

The paper is organized as follows. A model for observations is described in Section 2 . A corrected $T(q)$-likelihood estimator is presented in Section 3 . The strong consistency of the $T(q)$-likelihood estimator is proved in Section 4 . Concluding remarks are collected in Section 5. Section [6] contains two auxiliary results used in Section 4 .

2010 Mathematics Subject Classification. Primary 62J12.

Key words and phrases. Exponential structural model, measurement error, $T(q)$-likelihood estimator, corrected estimation function. 


\section{A MODEL FOR OBSERVATIONS}

Consider the response $y$ with the probability density $f(y \mid \lambda)=\lambda e^{-\lambda y}, y \geq 0$, and let $\xi$ be an unobservable random variable such that $\lambda=e^{\beta_{0}+\beta_{1} \xi}$. The variable $x=\xi+\delta$ is observed instead of the hidden variable $\xi$, where $\delta \sim \mathcal{N}\left(0, \sigma_{\delta}^{2}\right)$. The random variable $\delta$ is called a measurement error. We assume that $\delta$ is independent of both $\xi$ and $y$. The variance $\sigma_{\delta}^{2}$ of the variable $\delta$ is known.

Independent realizations of the model $z_{i}=\left(y_{i}, x_{i}\right), i=1, \ldots, n$, are observed. The aim is to estimate the vector $\beta=\left(\beta_{0} ; \beta_{1}\right)^{T}$ (here $\beta$ is the true value of the parameter). Put $f(y, \xi, \beta)=f(y \mid \lambda)$ and $\lambda=e^{\beta_{0}+\beta_{1} \xi}$. We assume that all exponential moments of the random variable $\xi$ are finite, that is $\mathrm{E} e^{a \xi}<\infty$ for all $a \in \mathbb{R}$. Then

$$
\begin{aligned}
& \mathrm{E}_{\beta} y=\mathrm{EE}_{\beta}(y \mid \xi)=\mathrm{E} \frac{1}{\lambda}=\mathrm{E} e^{-\beta_{0}-\beta_{1} \xi}=e^{-\beta_{0}} \mathrm{E}^{-\beta_{1} \xi}, \\
& \operatorname{Var}_{\beta} y=\mathrm{E}_{\operatorname{Var}_{\beta}}(y \mid \xi)+\operatorname{Var}_{\beta}(y \mid \xi)=\mathrm{E} \frac{1}{\lambda^{2}}+\operatorname{Var} \frac{1}{\lambda}=\mathrm{E} e^{-2 \beta_{0}-2 \beta_{1} \xi}+\operatorname{Var} e^{-\beta_{0}-\beta_{1} \xi} \\
& =e^{-2 \beta_{0}}\left(2 \mathrm{E} e^{-2 \beta_{1} \xi}-\left(\mathrm{E} e^{-\beta_{1} \xi}\right)^{2}\right) .
\end{aligned}
$$

Consider the Box-Cox transformation

$$
T(q, u)= \begin{cases}\frac{u^{1-q}-1}{1-q}, & \text { if } q \neq 1, \\ \ln u, & \text { if } q=1\end{cases}
$$

for $u>0$ and $q>0$.

A $T(q)$-likelihood estimating function is defined by

$$
S^{(q)}(y, \xi, \beta)=\frac{\partial}{\partial \beta} T(q, f(y, \xi, \beta))=f^{-q} \frac{\partial f}{\partial \beta}=e^{\lambda y(q-1)} \lambda^{1-q}(1-\lambda y)(1 ; \xi)^{T} .
$$

If $q=1$, then $S^{(q)}$ coincides with the estimating function of the maximum likelihood method. The function $S^{(q)}$ is considered in the papers [2, 3] for the case where the model does not involve measurement errors. If $q=q_{n} \rightarrow 1$ and $\sqrt{n}\left(q_{n}-1\right) \rightarrow 0$ as $n \rightarrow \infty$, then the $T(q)$-likelihood estimating function yields a consistent estimator $\beta$ which is as effective as the maximum likelihood estimator, but the behavior of the $T(q)$-likelihood estimator is nicer for small samples. We denote the maximum likelihood estimator by $\widehat{\beta}_{n}$. The estimator $\widehat{\beta}_{n}$ is given by

$$
\widehat{\beta}_{n}=\underset{\beta \in \Theta}{\operatorname{argmax}} \sum_{i=1}^{n} \ln \left(f\left(y_{i}, \xi_{i}, \beta\right)\right),
$$

where $\widehat{\beta}_{n}=\left(\widehat{\beta}_{0, n}, \widehat{\beta}_{1, n}\right)^{T}$ and the set of parameters $\Theta$ is a subset of $\mathbb{R}^{2}, \Theta \subset \mathbb{R}^{2}$.

\section{Construction of the estimating equation}

We modify the estimating function $S^{(q)}$ in order to consider models with measurement errors. For this, we construct a corrected estimating function $S_{C}^{(q)}$ such that

$$
\mathrm{E}_{b}\left[S_{C}^{(q)}(y, x, \beta) \mid y, \xi\right]=S^{(q)}(y, \xi, \beta)
$$

almost surely for all $\beta$ of $\Theta$. 
The corrected $T(q)$-likelihood estimator $\widehat{\beta}_{n}(q)$ is defined as a measurable solution of the equation

$$
\sum_{i=1}^{n} S_{C}^{(q)}\left(y_{i}, x_{i}, \beta\right)=0, \quad \beta \in \Theta .
$$

Put $h(y, x)=S_{C}^{(q)}(y, x, \beta)$. Then

$$
\begin{aligned}
\mathrm{E}_{b}[h(y, x) \mid y, \xi] & =f^{-q} \frac{\partial f}{\partial \beta}=e^{\lambda(q-1) y} \cdot\left(\lambda^{1-q}-\lambda^{2-q} y\right)\left(\begin{array}{l}
1 \\
\xi
\end{array}\right) \\
& =\left(\sum_{n=0}^{\infty} \frac{\lambda^{n-q+1}(q-1)^{n} y^{n}}{n !}-\sum_{n=0}^{\infty} \frac{\lambda^{n-q+2}(q-1)^{n} y^{n+1}}{n !}\right)\left(\begin{array}{l}
1 \\
\xi
\end{array}\right),
\end{aligned}
$$

where $\lambda=e^{\beta_{0}+\beta_{1} \xi}$. A solution of the deconvolution equation (3) can be found in the following form:

$$
h(y, x)=\sum_{n=0}^{\infty} \frac{(q-1)^{n} y^{n}}{n !}\left(\begin{array}{l}
\varphi_{n}(x) \\
\psi_{n}(x)
\end{array}\right)-\sum_{n=0}^{\infty} \frac{(q-1)^{n} y^{n+1}}{n !}\left(\begin{array}{l}
\varphi_{n+1}(x) \\
\psi_{n+1}(x)
\end{array}\right) .
$$

Here $\varphi_{n}(x)$ and $\psi_{n}(x)$ satisfy the deconvolution equations

$$
\begin{aligned}
\mathrm{E}\left[\varphi_{n}(x) \mid \xi\right]=\lambda^{n-q+1} & =e^{\left(\beta_{0}+\beta_{1} \xi\right)(n-q+1)}, \\
\mathrm{E}\left[\psi_{n}(x) \mid \xi\right]=\lambda^{n-q+1} \xi & =e^{\left(\beta_{0}+\beta_{1} \xi\right)(n-q+1)} \xi .
\end{aligned}
$$

First we solve equation (5). Let $a_{n}=\beta_{0}(n-q+1)$ and $r_{n}=\beta_{1}(n-q+1)$. Then we rewrite (5) with $a_{n}$ and $r_{n}$ as $\mathrm{E}\left[\varphi_{n}(x) \mid \xi\right]=e^{a_{n}+r_{n} \xi}$. We search for a solution $\varphi_{n}(x)$ such that $\varphi_{n}(x)=C_{n} e^{r_{n} x}$. For such a function $\varphi_{n}(x)$, we have

$$
\mathrm{E}\left(e^{r_{n}(\xi+\delta)} \cdot C_{n} \mid \xi\right)=C_{n} e^{r_{n} \xi} \mathrm{E} e^{r_{n} \delta}=e^{a_{n}+r_{n} \xi},
$$

whence $C_{n}=\left(\mathrm{E} e^{r_{n} \delta}\right)^{-1} e^{a_{n}}$. Recall that $\delta \sim \mathcal{N}\left(0, \sigma_{\delta}^{2}\right)$. The equality $\mathrm{E} \exp \left(r_{n} \delta\right)=$ $\exp \left(r_{n}^{2} \sigma_{\delta}^{2} / 2\right)$ implies that

$$
\mathrm{E} \delta \exp \left(r_{n} \delta\right)=\frac{d}{d r_{n}} \mathrm{E} \exp \left(r_{n} \delta\right)=r_{n} \sigma_{\delta}^{2} \exp \left(\frac{r_{n}^{2} \sigma_{\delta}^{2}}{2}\right)
$$

This means that

$$
\begin{aligned}
\varphi_{n}(x) & =C_{n} e^{r_{n} x}=\frac{e^{\left(\beta_{0}+\beta_{1} x\right)(n-q+1)}}{\mathrm{E} e^{\beta_{1}(n-q+1) \delta}} \\
& =\exp \left(\left(\beta_{0}+\beta_{1} x\right)(n-q+1)-\frac{\beta_{1}^{2} \sigma_{\delta}^{2}(n-q+1)^{2}}{2}\right) .
\end{aligned}
$$

Now we solve equation (6). Using the notation $a_{n}$ and $r_{n}$ introduced above, we rewrite equation (6) as

$$
\mathrm{E}\left[\psi_{n}(x) \mid \xi\right]=\xi e^{a_{n}+r_{n} \xi} .
$$

We search for a solution $\psi_{n}(x)$ such that $\psi_{n}(x)=e^{r_{n} x}\left(C_{1, n}+C_{2, n} x\right)$. Similarly to the reasoning above, we conclude that

$$
\begin{aligned}
\psi_{n}(x) & =e^{r_{n} x}\left(C_{1, n}+C_{2, n} x\right)=-\frac{e^{a_{n}+r_{n} x} \mathrm{E} \delta e^{r_{n} \delta}-x e^{a_{n}+r_{n} x} \mathrm{E} e^{r_{n} \delta}}{\left(\mathrm{E} e^{r_{n} \delta}\right)^{2}} \\
& =\frac{e^{\left(\beta_{0}+\beta_{1} x\right)(n-q+1)}}{\mathrm{E} e^{\beta_{1}(n-q+1) \delta}}\left(x-\frac{\mathrm{E} \delta e^{\beta_{1} \delta(n-q+1)}}{\mathrm{E} e^{\beta_{1} \delta(n-q+1)}}\right) \\
& =\left(x-\beta_{1}(n-q+1) \sigma_{\delta}^{2}\right) \exp \left(\left(\beta_{0}+\beta_{1} x\right)(n-q+1)-\frac{\beta_{1}^{2} \sigma_{\delta}^{2}(n-q+1)^{2}}{2}\right) .
\end{aligned}
$$


As a result, we get $h(y, x)$ from (44), (77), and (8). The series on the right-hand side of (4) converge absolutely by the d'Alembert ratio test. The order of summation can be changed by Fubini's theorem which allows us to evaluate $\mathrm{E}\left[\varphi_{n}(x) \mid \xi\right]$ and $\mathrm{E}\left[\psi_{n}(x) \mid \xi\right]$. This confirms that the function $h(y, x)$ defined by (4) with the functions $\varphi_{n}(x)$ and $\psi_{n}(x)$ given by (7) and (8), respectively, satisfies equation (1).

If $q=1$, then equality (4) implies that

$$
S_{C}^{(1)}(y, x, \beta)=\left(\begin{array}{l}
\varphi_{0} \\
\psi_{0}
\end{array}\right)-y\left(\begin{array}{l}
\varphi_{1} \\
\psi_{1}
\end{array}\right)=\left(\begin{array}{c}
1-y \exp \left(\beta_{0}+\beta_{1} x-\frac{\beta_{1}^{2} \sigma_{\delta}^{2}}{2}\right) \\
x-y\left(x-\beta_{1} \sigma_{\delta}^{2}\right) \exp \left(\beta_{0}+\beta_{1} x-\frac{\beta_{1}^{2} \sigma_{\delta}^{2}}{2}\right)
\end{array}\right) .
$$

Note that the distribution of $\xi$ does not matter for the construction of the estimator $\widehat{\beta_{n}}(q)$, $q>0$, described above.

\section{Consistency of the estimator}

Below we use the word "eventually" to abbreviate the following text. Let

$$
\left\{U_{n}: n \geq 1\right\}
$$

be a sequence of random variables and let $A_{n}\left(U_{n}\right)$ denote a statement about $U_{n}$. We say that a sequence of statements $A_{n}\left(U_{n}\right)$ holds eventually with respect to the probability measure $\mathrm{P}$ if there exists a random event $\Omega_{0} \subset \Omega$ such that $\mathrm{P}\left(\Omega_{0}\right)=1$ and, for all $\omega \in \Omega_{0}$, there exists a positive integer number $N(\omega)$ for which the statement $A_{n}\left(U_{n}(\omega)\right)$ holds for all $n \geq N$.

Theorem 4.1. Assume that

1. $q=q_{n}, 0<q_{n} \leq 1$ for all $n \geq 1$, and $q_{n} \rightarrow 1$ as $n \rightarrow \infty$;

2. the set of parameters $\Theta$ is a known compact subset of $\mathbb{R}^{2}$ and the true value $b$ of the parameter $\beta$ is an inner point of $\Theta$;

3. there exists $K>0$ such that $|\xi| \leq K$ almost surely, where $K$ is an unknown constant.

4. $\operatorname{Var} \xi \neq 0$.

Then equation (2) has a solution eventually.

Now we introduce the estimator $\widehat{\beta}_{C S}^{(q)}$ as a solution of equation (2) if such a solution exists; otherwise we write $\widehat{\beta}_{C S}^{(q)}=0$. The proof of Theorem 4.1 is given below.

Theorem 4.2. Let all the assumptions of Theorem 4.1 hold. Then the estimator $\widehat{\beta}_{C S}^{(q)}$ is strongly consistent, that is, $\widehat{\beta}_{C S}^{(q)} \rightarrow b$ with probability one as $n \rightarrow \infty$, where $b$ is the true value of the parameter $\beta$.

Proof of Theorems 4.1 and 4.2. We use Usol'tseva's lemma 6] (see Lemma 6.1 in Section 6). The estimating equation

$$
\sum_{i=1}^{n} S_{C}\left(y_{i}, x_{i}, \beta, q_{n}\right)=0
$$

is such that $q_{n} \rightarrow 1$ as $n \rightarrow \infty$, where

$$
\left(\begin{array}{l}
S_{1}\left(y_{i}, x_{i}, \beta, q_{n}\right) \\
S_{2}\left(y_{i}, x_{i}, \beta, q_{n}\right)
\end{array}\right)=S_{C}\left(y_{i}, x_{i}, \beta, q_{n}\right)=S_{C}^{\left(q_{n}\right)}\left(y_{i}, x_{i}, \beta\right)
$$


Write

$$
\begin{gathered}
S_{n}(\beta)=\frac{1}{n} \sum_{i=1}^{n} S_{C}\left(y_{i}, x_{i}, \beta, 1\right), \\
\Phi_{n}(\beta)=\frac{1}{n} \sum_{i=1}^{n}\left(S_{C}\left(y_{i}, x_{i}, \beta, q_{n}\right)-S_{C}\left(y_{i}, x_{i}, \beta, 1\right)\right)
\end{gathered}
$$

and rewrite the estimating equation as follows:

$$
S_{n}(\beta)+\Phi_{n}(\beta)=0, \quad \beta \in \Theta
$$

Then

$$
\begin{aligned}
& \mathrm{E}_{b} y^{n} e^{\beta_{1} \xi}=\mathrm{EE}_{b}\left(y^{n} e^{\beta_{1} \xi} \mid \xi\right)=\mathrm{E} \frac{e^{\beta_{1} \xi} n !}{\lambda^{n}\left(b_{0}, b_{1}\right)}=n ! e^{-b_{0} n} \mathrm{E}^{\left(\beta_{1}-b_{1} n\right) \xi}, \\
& \mathrm{E}_{b} y e^{\beta_{1} \xi}=\mathrm{E}_{b}\left(y e^{\beta_{1} \xi} \mid \xi\right)=\mathrm{E} \frac{e^{\beta_{1} \xi}}{\lambda\left(b_{0}, b_{1}\right)}=e^{-b_{0}} \mathrm{E} e^{\left(\beta_{1}-b_{1}\right) \xi}, \\
& \mathrm{E}_{b} y|\xi| e^{\beta_{1} \xi}=\mathrm{EE}_{b}\left(y|\xi| e^{\beta_{1} \xi} \mid \xi\right)=\mathrm{E} \frac{|\xi| e^{\beta_{1} \xi}}{\lambda\left(b_{0}, b_{1}\right)}=e^{-b_{0}} \mathrm{E}|\xi| e^{\left(\beta_{1}-b_{1}\right) \xi}, \\
& \mathrm{E}_{b} y \xi^{2} e^{\beta_{1} \xi}=\mathrm{EE}_{b}\left(y \xi^{2} e^{\beta_{1} \xi} \mid \xi\right)=\mathrm{E} \frac{\xi^{2} e^{\beta_{1} \xi}}{\lambda\left(b_{0}, b_{1}\right)}=e^{-b_{0}} \mathrm{E} \xi^{2} e^{\left(\beta_{1}-b_{1}\right) \xi}, \\
& \mathrm{E} e^{a x}=\mathrm{E} e^{a \xi+a \delta}=\mathrm{E} e^{a \xi} \exp \left\{\frac{a^{2} \sigma_{\delta}^{2}}{2}\right\}, \\
& \mathrm{E} x e^{a x}=\mathrm{E} \xi e^{a \xi} \mathrm{E} e^{a \delta}+\mathrm{E} e^{a \xi} \mathrm{E} \delta e^{a \delta}=\left(\mathrm{E} \xi e^{a \xi}+a \sigma_{\delta}^{2} \mathrm{E} e^{a \xi}\right) \exp \left\{\frac{a^{2} \sigma_{\delta}^{2}}{2}\right\} .
\end{aligned}
$$

Recall that $\delta \sim \mathcal{N}\left(0, \sigma_{\delta}^{2}\right)$. The equality $\mathrm{E} \exp \left(r_{n} \delta\right)=\exp \left(r_{n}^{2} \sigma_{\delta}^{2} / 2\right)$ implies that

$$
\mathrm{E} \delta^{2} \exp \left(r_{n} \delta\right)=\frac{d^{2}}{d r_{n}^{2}} \mathrm{E} \exp \left(r_{n} \delta\right)=\left(r_{n}^{2} \sigma_{\delta}^{4}+\sigma_{\delta}^{2}\right) \exp \left(\frac{r_{n}^{2} \sigma_{\delta}^{2}}{2}\right)
$$

Then

$$
\mathrm{E} x^{2} e^{a x}=\left(\mathrm{E} \xi^{2} e^{a \xi}+2 a \sigma_{\delta}^{2} \mathrm{E} \xi e^{a \xi}+\left(a^{2} \sigma_{\delta}^{4}+\sigma_{\delta}^{2}\right) \mathrm{E} e^{a \xi}\right) \exp \left(a^{2} \sigma_{\delta}^{2} / 2\right)
$$

The expectations on the right-hand side of the latter relation are finite by assumption 3 of Theorem 4.1 .

Consider assumption 1 of Lemma 6.1. The inclusion $S_{C}\left(y_{i}, x_{i}, \cdot, 1\right) \in C^{1}(\Theta)$ almost surely follows from the definition of the vector function $S_{C}\left(y_{i}, x_{i}, \cdot, 1\right)$ (we say that $S_{C}\left(y_{i}, x_{i}, \cdot, 1\right)$ belongs to the class $C^{1}(\Theta)$ if $S_{C}\left(y_{i}, x_{i}, \cdot, 1\right)$ is a continuously differentiable function in an open set containing $\Theta$ ).

We introduce a norm in $\mathbb{R}^{2}$ by $\|z\|=\left|z_{1}\right|+\left|z_{2}\right|, z \in \mathbb{R}^{2}$. Using the independence of the random variables $\xi$ and $\delta$ and the inequality $\mathrm{E}|\delta| e^{\beta_{1} \delta} \leq \sigma_{\delta} e^{\beta_{1}^{2} \sigma_{\delta}^{2}}$ that follows from the 
Cauchy-Schwartz inequality, we obtain the following bound for the expectation

$$
\begin{aligned}
\mathrm{E}_{b}\left\|S_{C}(y, x, \beta, 1)\right\|= & \mathrm{E}_{b}\left|1-y \exp \left(\beta_{0}+\beta_{1} \xi+\beta_{1} \delta-\frac{\beta_{1}^{2} \sigma_{\delta}^{2}}{2}\right)\right| \\
& +\mathrm{E}_{b}\left|\xi+\delta-y\left(\xi+\delta-\beta_{1} \sigma_{\delta}^{2}\right) \cdot \exp \left(\beta_{0}+\beta_{1} \xi+\beta_{1} \delta-\frac{\beta_{1}^{2} \sigma_{\delta}^{2}}{2}\right)\right| \\
\leq & 1+\exp \left(\beta_{0}-\frac{\beta_{1}^{2} \sigma_{\delta}^{2}}{2}\right) \mathrm{E} e^{\beta_{1} \delta} \mathrm{E}_{b} y e^{\beta_{1} \xi}+\mathrm{E}|\xi|+\mathrm{E}|\delta| \\
& +\exp \left(\beta_{0}-\frac{\beta_{1}^{2} \sigma_{\delta}^{2}}{2}\right)\left(\mathrm{E} e^{\beta_{1} \delta} \mathrm{E}_{b} y\left(|\xi|+\left|\beta_{1}\right| \sigma_{\delta}^{2}\right) e^{\beta_{1} \xi} \mathrm{E}|\delta| e^{\beta_{1} \delta} \mathrm{E}_{b} y e^{\beta_{1} \xi}\right) \\
\leq & 1+\mathrm{E}|\xi|+\mathrm{E}|\delta| \\
& +e^{\beta_{0}-b_{0}}\left(\mathrm{E}|\xi| e^{\left(\beta_{1}-b_{1}\right) \xi}+\left(1+\sigma_{\delta} \exp \frac{\beta_{1}^{2} \sigma_{\delta}^{2}}{2}+\left|\beta_{1}\right| \sigma_{\delta}^{2}\right) \mathrm{E}^{\left(\beta_{1}-b_{1}\right) \xi}\right),
\end{aligned}
$$

since the distribution of $\delta$ is normal. The terms on the right-hand side of this inequality are bounded from above by assumption 3 of Theorem 4.1. Thus,

$$
\mathrm{E}_{b}\left\|S_{C}(y, x, \beta, 1)\right\|<\infty .
$$

The function

$$
\begin{aligned}
S_{\infty}(\beta, b) & :=\mathrm{E}_{b} S_{C}(y, x, \beta, 1)=\mathrm{E}_{b} S_{C}^{(1)}(y, x, \beta)=\mathrm{E}_{b} \mathrm{E}_{b}\left(S^{(1)}(y, x, \beta) \mid y, \xi\right) \\
& =\mathrm{E}_{b} S^{(1)}(y, \xi, \beta)=\mathrm{E}_{b}(1-\lambda(\beta) y)(1 ; \xi)^{T}=\mathrm{E}\left(1-\frac{\lambda(\beta)}{\lambda(b)}\right)(1 ; \xi)^{T} \\
& =\mathrm{E}\left(1-\exp \left(\beta_{0}-b_{0}+\left(\beta_{1}-b_{1}\right) \xi\right)\right)(1 ; \xi)^{T}
\end{aligned}
$$

involved in assumption 2 of Lemma 6.1 is continuous with respect to $\beta=\left(\beta_{0} ; \beta_{1}\right)^{T}$ in the set $\Theta$.

Now we check assumption 3 of Lemma 6.1. The Jacobi matrix

$$
\frac{\partial S_{C}^{(1)}(y, x, \beta)}{\partial \beta^{T}}=y \exp \left(\beta_{0}+\beta_{1} x-\frac{\beta_{1}^{2} \sigma_{\delta}^{2}}{2}\right)\left(\begin{array}{cc}
-1 & -\left(x-\beta_{1} \sigma_{\delta}^{2}\right) \\
-\left(x-\beta_{1} \sigma_{\delta}^{2}\right) & \left(\sigma_{\delta}^{2}-\left(x-\beta_{1} \sigma_{\delta}^{2}\right)^{2}\right)
\end{array}\right)=\left(a_{i j}\right)_{i, j=1}^{2}
$$

is a $2 \times 2$ matrix in the cases under consideration.

Herein, we use the matrix norm

$$
\|A\|=\sum_{i, j=1}^{2}\left|a_{i, j}\right|
$$

The expectation of the norm of the matrix $\partial S_{C}^{(1)}(y, x, \beta) / \partial \beta^{T}$ is bounded from above by assumption 3 of Theorem 4.1 as follows:

$$
\begin{aligned}
\mathrm{E}_{b} \| & \frac{\partial S_{C}^{(1)}(y, x, \beta)}{\partial \beta^{T}} \| \\
\leq & \exp \left(\beta_{0}-\frac{\beta_{1}^{2} \sigma_{\delta}^{2}}{2}\right) \mathrm{E}_{b} y\left(1+2|x|+2\left|\beta_{1}\right| \sigma_{\delta}^{2}+\sigma_{\delta}^{2}+2 x^{2}+2 \beta_{1}^{2} \sigma_{\delta}^{4}\right) e^{\beta_{1} x} \\
\leq e^{\beta_{0}-b_{0}}\left(2 \mathrm{E}|\xi| e^{\left(\beta_{1}-b_{1}\right) \xi}\right. & \left.+\mathrm{E}^{2} e^{\left(\beta_{1}-b_{1}\right) \xi}\right)<\infty .
\end{aligned}
$$


Next we consider assumption 4 of Lemma 6.1. The Jacobi matrix

$$
\frac{\partial S_{\infty}(\beta, b)}{\partial \beta^{T}}=-e^{\beta_{0}-b_{0}}\left(\begin{array}{cc}
\mathrm{E} e^{\left(\beta_{1}-b_{1}\right) \xi} & \mathrm{E} \xi e^{\left(\beta_{1}-b_{1}\right) \xi} \\
\mathrm{E} \xi e^{\left(\beta_{1}-b_{1}\right) \xi} & \mathrm{E} \xi^{2} e^{\left(\beta_{1}-b_{1}\right) \xi}
\end{array}\right)
$$

is symmetric; the matrix

$$
V:=\left.\frac{\partial S_{\infty}(\beta, b)}{\partial \beta^{T}}\right|_{\beta=b}=\frac{\partial S_{\infty}(b, b)}{\partial \beta^{T}}
$$

is nonsingular, since its determinant is nonzero in view of assumption 4 of Theorem 4.1 ;

$$
\operatorname{det} \frac{\partial S_{\infty}(b, b)}{\partial \beta^{T}}=\left|\begin{array}{cc}
1 & \mathrm{E} \xi \\
\mathrm{E} \xi & \mathrm{E} \xi^{2}
\end{array}\right|=\operatorname{Var} \xi \neq 0 .
$$

To check assumption 5 of Lemma 6.1 we solve the following system of equations with respect to the unknown $\beta$ :

$$
S_{\infty}(\beta, b)=0
$$

This system reduces to

$$
\left\{\begin{array}{l}
1-e^{\beta_{0}-b_{0}} \mathrm{E} e^{\left(\beta_{1}-b_{1}\right) \xi}=0, \\
\mathrm{E} \xi-e^{\beta_{0}-b_{0}} \mathrm{E} \xi e^{\left(\beta_{1}-b_{1}\right) \xi}=0 .
\end{array}\right.
$$

If $\beta=b$, then $S_{\infty}(b, b)=0$.

Assume that there exists $\beta \neq b$ such that $S_{\infty}(\beta, b)=0$. Put $f(\beta)=S_{\infty}(\beta, b)$. The function $g(t)=(f(t b+(1-t) \beta), b-\beta)$ is such that $g(0)=g(1)=0$. According to Rolle's theorem, there exists $\tau \in(0,1)$ such that $g^{\prime}(\tau)=0$ and

$$
(b-\beta)^{T}\left(\left.\frac{\partial S_{\infty}(\beta, b)}{\partial \beta^{T}}\right|_{\beta=\bar{b}}\right)(b-\beta)=0,
$$

where the point $\bar{b}$ belongs to the interval whose end points are $b$ and $\beta$. The Cauchy inequality implies that

$$
\left(\mathrm{E} \xi e^{\left(\beta_{1}-b_{1}\right) \xi / 2} e^{\left(\beta_{1}-b_{1}\right) \xi / 2}\right)^{2} \leq \mathrm{E} \xi^{2} e^{\left(\beta_{1}-b_{1}\right) \xi} \mathrm{E} e^{\left(\beta_{1}-b_{1}\right) \xi}
$$

whence

$$
\operatorname{det} \frac{\partial S_{\infty}(\beta, b)}{\partial \beta^{T}} \geq 0
$$

In fact, this inequality is strict, since

$$
\frac{\xi e^{\left(\beta_{1}-b_{1}\right) \xi / 2}}{e^{\left(\beta_{1}-b_{1}\right) \xi / 2}}=\xi
$$

is not a constant in view of assumption 4 of Theorem 4.1. Applying Sylvester's criterion, we prove that the matrix

$$
\left.\frac{\partial S_{\infty}(\beta, b)}{\partial \beta^{T}}\right|_{\beta=\bar{b}}
$$

is negative definite for all $b \in \Theta$. This contradicts equality (10).

Therefore, the equation $S_{\infty}(\beta, b)=0$ has a unique root in $\Theta$. Moreover, $S_{\infty}(\beta, b)=0$ if and only if $\beta=b$.

Next we check assumption 6 of Lemma 6.1. In order to show that

$$
\sup _{\beta \in \Theta}\left|\Phi_{n}(\beta)\right| \stackrel{P 1}{\longrightarrow} 0
$$


we estimate

$$
\begin{aligned}
&\left\|\Phi_{n}(\beta)\right\| \leq \frac{1}{n} \sum_{i=1}^{n}\left\|S_{C}\left(y_{i}, x_{i}, \beta, q_{n}\right)-S_{C}\left(y_{i}, x_{i}, \beta, 1\right)\right\| \\
& \leq \frac{1}{n} \sum_{i=1}^{n} \sum_{k=1}^{2} \sup _{q_{n} \leq \gamma_{n} \leq 1, \beta \in \Theta}\left|\frac{\partial S_{k}\left(y_{i}, x_{i}, \beta, \gamma_{n}\right)}{\partial q}\right|\left|q_{n}-1\right|, \\
&\left|q_{n}-1\right| \cdot \frac{1}{n} \sum_{i=1}^{n} \sum_{k=1}^{2} \sup _{1-\tilde{\delta} \leq \gamma \leq 1, \beta \in \Theta}\left|\frac{\partial S_{k}\left(y_{i}, x_{i}, \beta, \gamma\right)}{\partial q}\right| \rightarrow 0, \quad n \rightarrow \infty .
\end{aligned}
$$

We require that, for $k=1,2$, there exists a number $\tilde{\delta}>0$ such that

$$
\mathrm{E}_{b} \sup _{1-\tilde{\delta} \leq \gamma \leq 1, \beta \in \Theta}\left|\frac{\partial S_{k}\left(y_{i}, x_{i}, \beta, \gamma\right)}{\partial q}\right|<\infty .
$$

Differentiating $S_{C}^{(q)}(y, x, \beta)$ with respect to $q$ we obtain the vector whose first component corresponding to the case of $k=1$ is such that

$$
\begin{aligned}
\frac{\partial S_{1}(y, x, \beta, q)}{\partial q}= & \sum_{n=0}^{\infty} \frac{(q-1)^{n} y^{n+1}}{n !} \exp \left(\left(\beta_{0}+\beta_{1} x\right)(n-q+2)-\frac{\beta_{1}^{2} \sigma_{\delta}^{2}(n-q+2)^{2}}{2}\right) \\
& +\sum_{n=0}^{\infty} \frac{(q-1)^{n} y^{n}}{n !}\left(-\beta_{0}-\beta_{1} x+\beta_{1}^{2} \sigma_{\delta}^{2}(n-q+1)\right) \\
& \quad \times \exp \left(\left(\beta_{0}+\beta_{1} x\right)(n-q+1)-\frac{\beta_{1}^{2} \sigma_{\delta}^{2}(n-q+1)^{2}}{2}\right) \\
& -\sum_{n=0}^{\infty} \frac{(q-1)^{n} y^{n+2}}{n !} \exp \left(\left(\beta_{0}+\beta_{1} x\right)(n-q+3)-\frac{\beta_{1}^{2} \sigma_{\delta}^{2}(n-q+3)^{2}}{2}\right) \\
& -\sum_{n=0}^{\infty} \frac{(q-1)^{n} y^{n+1}}{n !}\left(-\beta_{0}-\beta_{1} x+\beta_{1}^{2} \sigma_{\delta}^{2}(n-q+2)\right) \\
& \quad \times \exp \left(\left(\beta_{0}+\beta_{1} x\right)(n-q+2)-\frac{\beta_{1}^{2} \sigma_{\delta}^{2}(n-q+2)^{2}}{2}\right) .
\end{aligned}
$$

Since $x \leq|x| \leq|\xi|+|\delta|,\left|\beta_{0}\right| \leq C_{0}$, and $\left|\beta_{1}\right| \leq C_{1}$ we get

$$
\begin{aligned}
\mathrm{E} \sup _{\left|\beta_{1}\right| \leq C_{1}} \exp \left(-\frac{\beta_{1}^{2} \sigma_{\delta}^{2}(n-q+1)^{2}}{2}+\beta_{1}(n-q+1)|\delta|\right) \\
\leq \mathrm{E} \sup _{\left|\beta_{1}\right| \leq C_{1}} \exp \left(-\frac{\beta_{1}^{2} \sigma_{\delta}^{2}(n-q+1)^{2}}{2}+\beta_{1}(n-q+1) \delta\right) \\
\quad+\mathrm{E} \sup _{\left|\beta_{1}\right| \leq C_{1}} \exp \left(-\frac{\beta_{1}^{2} \sigma_{\delta}^{2}(n-q+1)^{2}}{2}-\beta_{1}(n-q+1) \delta\right) \\
\leq 2 \mathrm{E} \sup _{\left|\beta_{1}\right| \leq C_{1}} \exp \left(-\frac{\beta_{1}^{2} \sigma_{\delta}^{2}(n-q+1)^{2}}{2}+\left|\beta_{1}\right|(n-q+1) \delta\right) .
\end{aligned}
$$


Lemma 6.2 implies the inequalities

$$
\begin{gathered}
\mathrm{E} \sup _{\left|\beta_{1}\right| \leq C_{1}} \exp \left(-\frac{\beta_{1}^{2} \sigma_{\delta}^{2}(n-q+1)^{2}}{2}+\left|\beta_{1}\right|(n-q+1) \delta\right) \leq C_{1} \sigma_{\delta}(n-q+1) \sqrt{\frac{2}{\pi}}+1 \\
\mathrm{E} \sup _{\left|\beta_{1}\right| \leq C_{1}}|\delta| \exp \left(-\frac{\beta_{1}^{2} \sigma_{\delta}^{2}(n-q+1)^{2}}{2}+\left|\beta_{1}\right|(n-q+1) \delta\right) \\
\leq \frac{C_{1}^{2} \sigma_{\delta}^{3}(n-q+1)^{2}}{\sqrt{2 \pi}}+C_{1} \sigma_{\delta}^{2}(n-q+1)+\sigma_{\delta} \sqrt{\frac{2}{\pi}}
\end{gathered}
$$

since $\delta=\sigma_{\delta} \tau$ and $\tau \sim \mathcal{N}(0,1)$.

Equality (9) together with assumptions 2 and 3 of Theorem 4.1 imply that the expectation

$$
\mathrm{E}_{b} \sup _{1-\tilde{\delta} \leq q \leq 1, \beta \in \Theta}\left|\frac{\partial S_{1}(y, x, \beta, q)}{\partial q}\right|
$$

is finite. Choose a number $\tilde{\delta}$ such that

$$
\tilde{\delta}<\exp \left(b_{0}-\left|b_{1}\right| K-C_{0}-C_{1} K\right) .
$$

The second component

$$
\frac{\partial S_{2}(y, x, \beta, q)}{\partial q}
$$

is estimated similarly to the first component.

Finally, we check assumption 7 of Lemma 6.1.

Let $n_{0}$ be a number such that $q_{n} \geq 1-\tilde{\delta}$ for all $n \geq n_{0}$, where the number $\tilde{\delta}$ chosen above satisfies (11). Then

$$
\begin{aligned}
\sup _{n \geq 1} \sup _{\beta \in \Theta}\left\|\frac{\partial \Phi_{n}(\beta)}{\partial \beta^{T}}\right\| \leq & \sup _{n \geq n_{0}} \frac{1}{n} \sum_{i=1}^{n} \sup _{\beta \in \Theta ; 1-\tilde{\delta} \leq q \leq 1}\left\|\frac{\partial}{\partial \beta^{T}}\left(S_{C}\left(y_{i}, x_{i}, \beta, q\right)-S_{C}\left(y_{i}, x_{i}, \beta, 1\right)\right)\right\| \\
& +\sup _{n<n_{0}} \frac{1}{n} \sum_{i=1}^{n} \sup _{\beta \in \Theta}\left\|\frac{\partial}{\partial \beta^{T}}\left(S_{C}\left(y_{i}, x_{i}, \beta, q_{n}\right)-S_{C}\left(y_{i}, x_{i}, \beta, 1\right)\right)\right\| .
\end{aligned}
$$

The term

$$
\sup _{n<n_{0}} \frac{1}{n} \sum_{i=1}^{n} \sup _{\beta \in \Theta}\left\|\frac{\partial}{\partial \beta^{T}}\left(S_{C}\left(y_{i}, x_{i}, \beta, q_{n}\right)-S_{C}\left(y_{i}, x_{i}, \beta, 1\right)\right)\right\|
$$

is finite almost surely.

Consider

$$
\begin{aligned}
& \left(\frac{\partial}{\partial \beta^{T}} S_{C}(y, x, \beta, q)-\frac{\partial}{\partial \beta^{T}} S_{C}(y, x, \beta, 1)\right)_{11} \\
& \quad=\sum_{n=0}^{\infty} \frac{(n-q+1)(q-1)^{n} y^{n}}{n !} \exp \left(\left(\beta_{0}+\beta_{1} x\right)(n-q+1)-\frac{\beta_{1}^{2} \sigma_{\delta}^{2}(n-q+1)^{2}}{2}\right) \\
& \quad-\sum_{n=0}^{\infty} \frac{(n-q+2)(q-1)^{n} y^{n+1}}{n !} \exp \left(\left(\beta_{0}+\beta_{1} x\right)(n-q+2)-\frac{\beta_{1}^{2} \sigma_{\delta}^{2}(n-q+2)^{2}}{2}\right) \\
& \quad+y \exp \left(\beta_{0}+\beta_{1} x-\frac{\beta_{1}^{2} \sigma_{\delta}^{2}}{2}\right) .
\end{aligned}
$$

Reasoning as in the proof of assumption 6 of Lemma 6.1, we conclude that the expectation is finite. The true value of the parameter is the same as that presented in the expression for $\lambda$. 
It is easy to check that

$$
\mathrm{E} \sup _{\beta \in \Theta, 1-\tilde{\delta} \leq q \leq 1}\left|\left(\frac{\partial}{\partial \beta^{T}} S_{C}\left(y_{i}, x_{i}, \beta, q\right)-\frac{\partial}{\partial \beta^{T}} S_{C}\left(y_{i}, x_{i}, \beta, 1\right)\right)_{k l}\right|<\infty
$$

for all $k, l=1,2$, where

$$
\left(\frac{\partial}{\partial \beta^{T}} S_{C}\left(y_{i}, x_{i}, \beta, q\right)-\frac{\partial}{\partial \beta^{T}} S_{C}\left(y_{i}, x_{i}, \beta, 1\right)\right)_{k l}
$$

is the entry $(k, l)$ of a $2 \times 2$ matrix. This implies that

$$
\sup _{n \geq 1} \sup _{\beta \in \Theta}\left\|\frac{\partial \Phi_{n}(\beta)}{\partial \beta^{T}}\right\|<\infty \quad \text { a.s. }
$$

Therefore, all the assumptions of Lemma 6.1 are checked and thus Theorems 4.1 and 4.2 hold by Lemma 6.1

\section{Concluding Remarks}

We studied the exponential structural model with normal measurement error. It is assumed that the variance $\sigma_{\delta}^{2}$ of the measurement error is known. To estimate an unknown parameter $b=\left(b_{0} ; b_{1}\right)^{T}$, we construct a corrected $T(q)$-likelihood estimator. Some sufficient conditions are given for this estimator to be strongly consistent.

\section{Appendix}

Lemma 6.1 ([6]). Let $(\Omega, \mathcal{F}, \mathrm{P})$ be a probability space and let $\Theta$ be a compact set in $\mathbb{R}^{m}$. Assume that independent identically distributed random vectors $Z_{i}, i=1, \ldots, n$, are observed in $\mathbb{R}^{k}$ and that their distribution depends on a parameter $\beta \in \Theta$. Given a Borel function $q: \Theta \times \mathbb{R}^{k} \rightarrow \mathbb{R}^{m}$ consider $S_{n}(\beta)=\frac{1}{n} \sum_{i=1}^{n} q\left(\beta, Z_{i}\right), \beta \in \Theta$. Let the true value of the parameter $\beta$ be equal to $b$ and let $b$ be an inner point of $\Theta$.

Assume further that:

1. $q(\cdot, Z) \in C^{1}(\Theta)$ almost surely; $\mathrm{E}_{b}\|q(\beta, Z)\|<\infty$ for $\beta \in \Theta$.

2. The function $S_{\infty}(\beta, b):=\mathrm{E}_{b} q(\beta, Z)$ is continuous with respect to $\beta$ in $\Theta$.

3. $\mathrm{E}_{b}\left\|\partial q(\beta, Z) / \partial \beta^{T}\right\|<\infty, \beta \in \Theta$.

4. $V:=\partial S_{\infty}(\beta, b) /\left.\partial \beta^{T}\right|_{\beta=b}$ is a nonsingular matrix.

5. $S_{\infty}(\beta, b)=0, \beta \in \Theta$, if and only if $\beta=b$.

Let random vector-valued functions $\Phi_{n}(\beta)=\Phi_{n}(\beta, \omega), n \geq 1$, assuming values in $\mathbb{R}^{m}$ be such that:

6. For all $\beta \in \Theta, \Phi_{n}(\beta) \rightarrow 0$ with probability one as $n \rightarrow \infty$, and $\Phi_{n}(\cdot) \in C^{1}(\Theta)$ almost surely for all $n \geq 1$.

7. $\sup _{n \geq 1} \sup _{\beta \in \Theta}\left\|\partial \Phi_{n}(\beta) / \partial \beta^{T}\right\|<\infty$ almost surely.

Then

a) eventually there exists a solution of the estimating equation $S_{n}(\beta)+\Phi_{n}(\beta)=0$, $\beta \in \Theta$

b) the estimator $\widehat{\beta}_{n}$ of the parameter $\beta$ for which $S_{n}\left(\widehat{\beta}_{n}\right)+\Phi_{n}\left(\widehat{\beta}_{n}\right)=0$ eventually is strongly consistent.

Lemma 6.2. Let $\tau \sim \mathcal{N}(0,1)$. Then

$$
\begin{gathered}
\mathrm{E} \sup _{|b| \leq C} \exp \left(-\frac{b^{2}}{2}+b \tau\right)=C \sqrt{\frac{2}{\pi}}+1, \\
\mathrm{E} \sup _{|b| \leq C}|\tau| \exp \left(-\frac{b^{2}}{2}+b \tau\right)=\frac{C^{2}}{\sqrt{2 \pi}}+C+\sqrt{\frac{2}{\pi}} .
\end{gathered}
$$


Proof of Lemma 6.2. If $|t| \leq C$, then

$$
\sup _{|b| \leq C} \exp \left(-\frac{b^{2}}{2}+b t\right)
$$

is attained at $b=t$. The same supremum is attained at $|b|=C$ if $|t| \geq C$. Consider

$$
\begin{aligned}
\text { E } \sup _{|b| \leq C} & \exp \left(-\frac{b^{2}}{2}+b \tau\right)=\frac{1}{\sqrt{2 \pi}} \int_{-\infty}^{\infty} \exp \left(-\frac{t^{2}}{2}\right) \sup _{|b| \leq C} \exp \left(-\frac{b^{2}}{2}+b t\right) d t \\
= & \frac{1}{\sqrt{2 \pi}} \int_{-C}^{C} \exp \left(-\frac{t^{2}}{2}\right) \sup _{|b| \leq C} \exp \left(-\frac{b^{2}}{2}+b t\right) d t \\
& +\frac{1}{\sqrt{2 \pi}} \int_{-\infty}^{-C} \exp \left(-\frac{t^{2}}{2}\right) \sup _{|b| \leq C} \exp \left(-\frac{b^{2}}{2}+b t\right) d t \\
& +\frac{1}{\sqrt{2 \pi}} \int_{C}^{\infty} \exp \left(-\frac{t^{2}}{2}\right) \sup _{|b| \leq C} \exp \left(-\frac{b^{2}}{2}+b t\right) d t \\
= & \frac{1}{\sqrt{2 \pi}} \int_{-C}^{C} \exp \left(-\frac{t^{2}}{2}-\frac{t^{2}}{2}+t^{2}\right) d t+\frac{1}{\sqrt{2 \pi}} \int_{-\infty}^{-C} \exp \left(-\frac{t^{2}}{2}-\frac{C^{2}}{2}-C t\right) d t \\
& +\frac{1}{\sqrt{2 \pi}} \int_{C}^{\infty} \exp \left(-\frac{t^{2}}{2}-\frac{C^{2}}{2}+C t\right) d t \\
= & C \sqrt{\frac{2}{\pi}}+1 .
\end{aligned}
$$

The second equality of Lemma 6.2 is proved by similar reasoning.

\section{ACKNOWLEDGMENT}

The author is grateful to Professor Kukush for the setting of the problem and fruitful discussions.

\section{BIBLIOGRAPHY}

1. C.-L. Cheng and H. Schneeweiss, Polynomial regression with errors in the variables, J. R. Stat. Society B 60 (1998), 189-199. MR 1625632

2. D. Ferrari and Y. Yang, Maximum Lq-likelihood estimation, Ann. Statist. 38 (2010), 753-783. MR2604695 (2011c:62069)

3. N. Kolev, Maximum T(q)-Likelihood Estimation: a New Method and its Application in Risk Management, $6^{\text {th }}$ Conference in Actuarial Science \& Finance on Samos, 2010, p. 22.

4. A. Kukush and H. Schneeweiss, Comparing different estimators in a non-linear measurement error model, I. Math. Methods Statist. 14 (2005), 53-79. MR2158071 (2006j:62068a)

5. A. Kukush,I. Markovsky, and S. Van Huffel, Consistent adjusted least squares estimator for errors-in-variables model $A X B=C$, Metrika 57 (2003), 253-285. MR1986189(2004d:62098)

6. O. S. Usol'tseva, A consistent estimator in the accelerated failure time model with censored observations and measurement errors, Teor. Imovir. Matem. Statyst. 82 (2010), 156-162; English transl. in Theory Probab. Math. Stat. 82 (2011), 161-169. MR2790491 (2011m:62346)

Department of Mathematical Analysis, Faculty for Mathematics and Mechanics, National Taras Shevchenko University, Volodymyrs'Ka Street, 64, Kyiv 01601, Ukraine

E-mail address: nebulous@bigmir.net

Received 02/JUNE/2011

Translated by S. V. KVASKO 